\title{
Ambivalence and obesity stigma in decisions about weight management: A qualitative study
}

\author{
Ian Brown*, Alex McClimens
}

Centre for Health \& Social Care Research, Sheffield Hallam University, Sheffield, UK;

*Corresponding Author: ian.brown@shu.ac.uk

Received 14 November 2012; revised 18 December 2012; accepted 22 December 2012

\section{ABSTRACT}

Background: Many adults do not take up weight management interventions even after apparently deciding to do so. Further research about decision making prior to the intervention would be useful. This paper presents a qualitative study exploring the process of decision making and the influences of obesity stigma. Methods: A pragmatic qualitative methodology, conducting indepth interviews with $\mathbf{5 2}$ participants all with BMI $>30 \mathrm{~kg} / \mathrm{m}^{2}$ and experience of efforts at weight management. Equal numbers of men and women with mean age $\mathbf{5 6 . 9}$ years completed interviews. Inductive analyses proceeded through systematic steps over a series of iterations. Findings: Decision making is difficult in the context of on-going mixed feelings over a long time. Thoughts and feelings become ingrained with habits and it is hard to separate out what is needed to think through a good decision. Thinking about weight brings a large volume of thoughts and feelings and apparent options or action choices. The volume of thoughts makes decisions difficult but, in the context of obesity stigma, many of the thoughts are negative. A variable sensitivity to these stigma-related thoughts adds further ambivalence and inhibition for taking decisions. The need for further thinking does not stand out in the context of the emotional resolving of thoughts about personal responsibility arising from obesity stigma. Conclusions: Obesity stigma contributes to a deeper ambivalence in the decision process and hence difficulty in decision making about weight management. Decision aid interventions and training of health care staff in communication skills for shared decision making are needed.

Keywords: Obesity; Stigma; Qualitative Research

\section{INTRODUCTION}

Obesity is a leading public health problem in many parts of the world affecting hundreds of millions of people [1]. In countries at the forefront of the pandemic a third or more of adults may soon have sufficient excess body fat to be at risk of health problems [2]. Among strategies to tackle obesity many health systems are investing in weight management interventions [3-8]. Clinical guidelines condensing the complex evidence in this field set out a range of dietary, physical activity, behavioral and psychological, interventions as a first step to help patients with weight loss [5-8]. Various mixes of all these elements are recommended, labeled broadly as behavioral and lifestyle interventions. However, healthy weight maintenance is difficult even with intensive and sustained lifestyle intervention input $[9,10]$. For many adults obesity becomes a long term condition requiring sustained motivation and engagement for successful management [11].

Given the evidence of a complex, difficult to manage, condition it is, therefore, unfortunate that in most cultures the popular assumption is that weight is readily under personal control $[12,13]$. Even among health professionals weight problems are perceived as a matter of a failure of personal effort in the face of simple lifestyle choices [14-16]. These views along with a range of deep rooted negative stereotypes are understood as a facet of the particular stigma attached to obesity [14]. Stigma is the phenomenon in which societal evaluations negatively impact on individuals' sense of identity and self-presentation in social interactions [15]. In many societies obesity brings a strongly devalued physical and moral identity, creating challenges for those affected by obesity in managing their identity and in presenting themselves in social interaction. Obesity stigma has general consequences in many spheres of life for affected individuals, including decision making about weight management interventions.

Within the evidence about effective interventions it is also apparent that recruitment and retention of participants in weight management programs is a problem [11- 
13,17]. Many adults do not take up lifestyle interventions even after apparently deciding to do so, or if enrolled, drop-out after a short time. Whilst this problem can be viewed from various perspectives, it is usually seen as one of personal motivation within interventions. Certainly the main thrust of clinical guidelines is on individual psychological theories of readiness to change and beliefs, attitudes and motivations in relation to health. Less attention is given to the decision making process prior to the intervention or to the impact of obesity stigma. This paper presents a qualitative study aiming to explore the process of decision making and the influences on this of aspects of obesity stigma. This was part of a larger study exploring influences on decisions about weight management.

\section{METHODS}

\subsection{Design and Recruitment}

The study employed a pragmatic qualitative methodology, now established in health research [18-21], aiming to illuminate the experiences and perceptions of people with obesity in relation to our research questions. Following UK National Health Service (NHS) Ethics approval participants were recruited from a pool of respondents to a regional postal survey about weight and health in 2012 [22]. All these respondents had agreed to being contacted further and also all had a BMI $>30 \mathrm{~kg} / \mathrm{m}^{2}$ indicating obesity. A total of 758 adults were added to a new sampling frame and stratified to gender and to socio-economic status. Stratification was employed because previous qualitative research in this field is heavily biased towards women rather than men and to those from more affluent backgrounds [23]. Potential participants were then randomly selected with attention to an even representation of gender and social backgrounds. An invitation to participate in an interview was sent to 128 individuals initially, about half responded expressing interest, and over a period of six weeks 52 volunteers completed interviews. A judgment was reached that data saturation had been achieved and no further recruitment was attempted.

\subsection{Participants}

Participants ranged in age from 26 years to 79 years with a mean age of 56.9 years $(n=52$, std dev $=12.2$ years). Participants were evenly distributed between men (50\%) and women (50\%), and between upper and lower income brackets. No volunteers dropped out of the study. There were no statistical differences in mean ages between gender and income groups.

\subsection{Data Collection}

The interview guide, with illustrative examples of ques- tioning probes, is shown in Box 1. Excluding preliminary opening dialogue oriented to establishing a good rapport the interviews ranged between 10 - 40 minutes with a mean of just under 20 minutes. There were no significant differences in interview length between the different groups of participants.

Telephone interviews were undertaken by experienced obesity research interviewers at a time convenient to the volunteer. A telephone, rather than face-to-face, interview meant it was easier to include more people and with more varied backgrounds than is typical in this type of study [23]. It also eliminated the potential, but unknown, effects of interviewer body size on data collection that might arise from face to face interaction.

The telephone interview data quality was checked by a comparison with the volume and richness of data produced from face to face interviews in previous studies about adult obesity [24,25]. In comparison to face to face interviews the exchanges were more focused and faster paced leading to a shorter interview time for comparable data but no inhibition apparent in discussing personal experiences.

\subsection{Data Analysis}

Interviews were transcribed verbatim and anonymised before entering into the QSR NVivo computer program to facilitate management [26]. Analyses proceeded through systematic steps over a series of iterations to initially develop and evaluate a coding framework grounded in the data $[19,26,27]$. All interviews were then fully coded by one author and coding checked by the other.

Outline of interview guide with illustrative questions/probes*:

Previous (recent) weight management actions

Can you think of a time you have done something about your weight? Please tell us about your most recent experience.

Encourage to recall and describe actions.

\section{Deciding processes for actions}

Looking back, just before you had actually done any of this, do you feel there was a clear point you decided what you were going to do? Did you feel in your mind that you were reaching a decision? Tell us more about the process of reaching a decision.

\section{Influences on decision making}

We'd like to explore a bit more about your thinking at the time just before taking action about your weight. What influenced your thoughts? Anything at all is relevant here.

Exploring key influences...

When you came to make a decision what had the most influence on you?

*Please note interviewers were encouraged to maintain a good rapport, covering topic areas in depth but flexibly

Box 1. Interview guide. 
Differences were resolved at team meetings. Data matrices, summaries and case stories were employed to facilitate analyses with further checking for instances that did not fit the framework or helped refine understanding of experiences and perceptions.

\section{RESULTS}

All participants could recall actions to manage weight at some time as an adult; half of participants were currently or recently (last 6 months) actively trying to lose weight. For most a mix of lifestyle actions had been followed to try to lose weight. Actions around foods and diet were most frequently talked about but also actions for more physical activity. A very few reported taking medicines or meal supplements or of being under recent care for surgery. The focus in this paper is on the decision process and the influence of obesity stigma. Quotations are followed by abbreviations to provide the gender $(\mathrm{M}=$ male, $\mathrm{F}=$ female), social background ( $\mathrm{U}=$ upper income group, $\mathrm{L}=$ lower income group) with interview identifier and followed by the participant's age in years.

\subsection{Decision Making}

Very few participants could recall a clear single point of decision making about weight management. Two exceptions were men thinking back to how heart problems dramatically triggered their awareness of their weight as a problem. However, the far more typical picture was a background awareness of weight as a problem that had been naggingly present for a long time. This was a vague continuing sense of weight as a problem, sometimes in the background sometimes in the foreground, but not absent. Linked to this was a similarly vague sense of what should be done about weight. In this context there was not a perception of taking conscious decisions about what to do or a clear plan being available.

I don't think it was ever achieved in you know conscious "okay its time to start doing this now". [M/U-021 age 40 years]

I've never really been happy with me weight. [...] it's a vicious circle that you can get into the only thing is you've really got to want to do it for yourself. [W/U-022 age 46]

I guess its probably I always knew it but never actually um made the decision to actually do something about it. [M/L-012 age 26]

There isn't a day that goes by if I'm honest that I don't think about it. [W/L-011 age 61]

For these participants there was a fluctuating ambivalence (mixed feelings) about size with awareness of a need for action heightened in certain contexts and by various triggers. Grappling with a personal response to this is a feature of ambivalence about weight control steps.
It's all contradictory really a lot of the feelings and thoughts I have were contradictory. [W/U-017 age 53]

Getting past this ambivalence to an acceptance that there is a responsibility to take action was felt to be important in a decision process. Indeed, reaching a settled state of mind was considered by some as a decision. However, it should be emphasized that this was not a thorough cognitive process but more about reaching an emotional resolution in response to thoughts about personal responsibility. The heightening of these kind of thoughts arise from the particular stigma linked to obesity.

In recalling the decision process participants found it hard to identify a separate (new) thought process about weight. Their perception was of an on-going nagging of the self not a new event requiring a decision. Indeed some struggled with the concept of a decision as such and the implication that they may have consciously thought through what to do. The sense was more of general feelings arising that it was necessary to do something but not of deciding what to do.

I'd say it's just its not a sort of you know a decision making process made of separate steps really it's a sort of its a way of life really [...] I don't see it as these sort of definite turning points you seem to be implying; really it's a way of life, keeping control of your weight for me. [W/U-023 age 62]

Believe it or not I've got 18 years of weekly weight records. Um but to be honest um it no I haven't I've had sporadic kind of you know just cut down a bit on this and that but no specific plans as such. [M/U-013 age 66]

These quotes suggest a chronic engagement with weight as a problem but not a thorough discrete thinking process as to what to do about it. An emotional response rather than a cognitive decision based on evaluation of salient information. It appears that as weight is bound into identity and habits over a long period it is harder to recognize the thinking required to decide what to do.

\subsection{Contexts and Triggers}

Overall, for most participants a mix of triggers and contexts set off thoughts about weight, sometimes repeated over and over:

I mean I've done it lots of times and it never seems to be the same thing but then I you know it never seems to be the same thing, that does you know that does make it. [...] I think it sometimes can be a health thing sometimes, can be other members of the family. Sometimes it can be just that you see yourself in the mirror and think "oh that's not me that's not good I want to be ...” [W/L-020 age 55]

Health reasons were the most frequently mentioned trigger. Also important was anticipation of social interactions in which a heightened sense of stigma would be 
likely to be experienced. Frequently participants recalled internal negative thoughts in anticipation of a social event like a wedding or holiday as a trigger for thoughts about weight control.

\subsection{Right Frame of Mind}

The "right frame of mind" was mentioned by many participants as a notable feature in their decision process. Various terms were used such as: "come to terms with"; "believe it for yourself”; “do it for yourself”; "mentally ready" among others. The concept was of reaching an emotional state (more feelings than thoughts) of acceptance of the personal responsibility involved for weight management. Note, though, in the quotes below, how this "right frame of mind" has a social relational dimensionfor example, “you're an individual” and "you may get pressure from some quarters, especially medical quarters”.

I personally think you know it's something that you've got to come to terms with and say you know this is what I want to do [...] because lots of people can talk to you about it say it's a good idea to do it unless you actually believe yourself that it is a good idea for you you're not going to do it anyway. [M/U-014 age 55]

You've got to be in the right frame of mind to actually want to lose some [M/U-020 age 46]

You're an individual and every person knows deep in their mind they know what works for them you know [W/L-021 age 26]

I mean everybody's different I'm not happy with my weight but you know I would like to lose weight but for me not anyone else. [W/L-18 age 43]

And it's taken I suppose about 18 months perhaps a little less to really sort of think it through and make the decision cause nobody else can do it for you and even though you may get pressure from some quarters especially medical quarters that you have to do it for yourself you can't do it for anyone else. [W/L-022 age 65]

However, this settled emotional state is not a decision about what to do and does not necessarily translate into action:

I did reach a decision that really I ought to do something but I was a bit wishy-washy about actually doing something (laughs). [...] The decision was made but what do they say the heart was willing but the flesh was weak so I didn't really pursue it very well. [...] I just realised that I was putting weight on and it was getting um more than I wanted and that it would be a good idea to do something about it and it was as easy as that. [W/U-013 age 61]

Some participants recalled a lack of clear information at this point to help them to decide what to do.

I mean I was conscious of it, the thing that puzzled me about it was all the information you kept getting seemed to contradict each other if you know what I mean? [...] I was getting all confused when I was asking for information. [M/L-013 age age 60]

I suppose in the last year to 18 months I really sort of got I really ought to try and do something but it's just finding out what I can actually do... [W/L-012 age 64]

\subsection{Influence of Obesity Stigma}

The presentation now focuses more directly on the effects of obesity stigma on participants' thoughts in relation to decisions about weight management. Most participants were more or less conscious of being influenced by wider cultural norms about body size, social roles and obesity. Generally this was the expectation that a slim build was approved and to be overweight was not acceptable. Television was the most frequently mentioned media influence in this respect.

I suppose you know the amount of exposure I get there must have been some influence to male figures that get to thrust at you same as women get influenced by you know fashion figures and things like that that get promoted on the television. [M/U-015 age 54]

I get to the point where it just depresses me feeling that I am overweight and you know it's what you see on the television it's what you see on magazines it's what you see around you. [W/U-017 age 53]

These general cultural influences brought a particular interpretation of expectations about roles and body size and about appropriate actions for weight control. The influence on thoughts, for example, as to what is appropriate for a man or woman of my age and with my kind of responsibilities or job to be doing about their weight.

I were determined not to get ridiculously thin either and I've not achieved that but because I don't like you look at people and they're so thin at my age. [W/U-019 age 51]

Awareness of a wider negative evaluation from others was an important influence on thoughts of a minority of participants across all groups; and one that evoked strong feelings (Box 2). Within these quotes are a range of negative stereotypes and associations, including greed and laziness, and apparent impact on emotional states, including shame, depression and embarrassment. For a few participants such stigma related thoughts provided a stimulus to make change and were seen as an acceptable social pressure. More typically, though, they were not viewed as helpful and were seen as being counter-productive for thinking clearly about health actions. Some participants were more sensitive to obesity stigma and found it upsetting and inhibiting. In general it added to ambivalence and difficulties in deciding what to do about weight control. 


\begin{abstract}
Stigma awareness at decision points:
There'd been a you know the banter at work was getting a bit much to deal with. [...] I was the um the fat boy in the office sort of thing. [M/U-015 age 54]

Well I'm ashamed really. I feel ashamed that I let myself get to the state that I'm at you know and I just I'm fat you know. [W/U-020 age 66]

I didn't want to be known as the fat mum at the school gates. [W/U-012 age 34]

I was a damn sight bigger than them so I did tend to think that people was looking at me. "Oh God look at her look at how fat she is" or, you know what I mean, never really accepted. If you see a fat person first thing you say, "She must be right greedy her". [W/L-019 age 62]
\end{abstract}

\section{Impact of stigma thoughts:}

A lad sort of commented he said "you're really big aren't you?" you know you don't want to hear things like that so it sort of just brings it to your forefront if you like you're suddenly aware of what people are thinking $[. .$.$] when someone makes a comment like that$ if anything its, um what's come into mind it depresses me only thing I want to go and do is get some biscuits [M/L-021 age 39]. I'm embarrassed a little bit you know not about what other people think, about what I think about meself. [M/L-022 age 41]

You feel you feel like a failure when you're overweight [...] you feel that people are judgmental about your weight even if they don't say anything. [W/U-011 age 53]

Oh actually it did the opposite because my doctor's call it a fat club I refuse to go (laughs) I didn't think it were very positive way of getting people who were overweight to go be weighed and get advice so no I didn't take it up to be honest. [W/U-019 age 51]

It just makes it makes it worse really it makes you feel um it's hard to explain really it doesn't give me the incentive to do it, it just makes it more difficult to you know to sort of come out and do something about it you feel you know embarrassed and ashamed I do find it yeah its difficult. [W/L-020 age 55]

You know it's like pointing the finger and saying "it's your fault" and I, it's very hard to put into words but it just makes me very cross really instead of encouraging people it's the opposite side of the coin its very negative and other people jump on the band wagon and start to say the same words which they perhaps never thought of before. [W/L-022 age 65]

Box 2. Stigma and decision making.

\section{DISCUSSION}

\subsection{Study Summary}

The study was successful in collecting rich qualitative data from men and women from different socio-economic backgrounds but, it should be noted, two thirds of the sample comprised adults aged 40 - 65 years. The study contributes to a deeper understanding of why decisions about weight management can be difficult and how they might be improved. It provides a potentially generalizable theory of relationships between phenomena salient to weight management; it does not provide a representative quantification of the issues identified. The findings are transferable, therefore, as qualitative research, to other contexts in which adults are taking decisions about weight control within cultures in which obesity is stigmatized. A number of key insights can be summarized before discussion of the wider literature.

First, decision making is difficult in the context of ongoing mixed feelings over a long time. Thoughts and feelings become ingrained with habits and it is hard to separate out what is needed to think through a good decision. This is typical of obesity as a long term chronic condition lacking a simple management intervention. Second, decision making is difficult because the need for "thinking through" does not stand out in the context of the emotional resolving of thoughts about personal responsibility. Again this is a heightened feature within weight decisions because of obesity stigma. Third, for many people, thinking about weight brings a large volume of thoughts and feelings and apparent options or action choices. Simply the volume of thoughts makes decisions difficult but, particularly in the context of obesity stigma, many of the thoughts and feelings are very negative. Fourth, there is a variable sensitivity to obesity stigma thoughts that can add further ambivalence and inhibition for taking decisions.

\subsection{Comparison with Literature}

The contours and impact of obesity stigma have been explored and described in much other research [15]. A review of qualitative studies undertaken in various, mainly urban, settings around the world found a majority reported obesity stigma [23]. This includes studies in Australia, UK and USA where obesity stigma is very pronounced [28-31]. Equally, many quantitative studies have also described the extent and impact of negative stereotypes and attitudes associated with obesity stigma [32,33]. Obesity stigma, as found in our study, is understood as a very deep rooted phenomenon in Western cultures. A substantial body of research demonstrates that the particularly negative attitudes about obesity are underpinned by beliefs about personal responsibility and that weight loss is readily under personal control. Negative stereotypes about body size and obesity are extremely deep rooted and powerful. Overall it is a world of mixed messages and pressures about body size. These influences make thinking clearly about weight management a challenge. It should also be noted here that recent research suggests an association between obesity and reduced cognitive function [34].

The reasons why people take actions about health has been a topic for research and debate over many years [35]; but the conceptualization and emphasis given to obesity stigma is an issue. Two prominent models will be considered briefly in discussion of our findings. These models are especially pertinent because they are widely included 
in the training of health professionals and in clinical guidelines [5-8]. The "Stages of Change" model remains influential despite a weak evidence base [36,37]. Broadly, as applied to obesity, the model suggests people would begin by not seeing weight as a problem (Pre-contemplation). They would then move on to become aware of it as a problem (Contemplation) and move on to take action for change that may or may not be maintained. Our findings do not support this view of stages or a cycle. Rather the typical picture is of on-going fluctuating levels of ambivalence punctuated by triggers and contexts that heighten awareness of weight thoughts. Furthermore, the relational psycho-social concept of stigma is difficult to fit within this individual readiness model.

Other widely influential psychological models suggest that actions arise from beliefs. For example, the "Theory of Planned Behavior" highlights the importance of beliefs about consequences of actions, about expectations of what others may think and about perceptions of the control we have over actions [38]. A number of personal, demographic and environmental background factors are seen to influence these beliefs. These models have better underpinning evidence [39]. Our findings fit this model better but nevertheless draw attention to factors, important for obesity, that are not sufficiently apparent within a generic psychological beliefs model. First, our findings highlight the difficulties of disentangling beliefs from identity, on-going habits and experiences in relation to weight and body size. Second, our findings highlight the distinct psychosocial pressures associated with body size and obesity stigma. Essentially stigma is a social phenomenon rather than a psychological belief. Social interactions are shot through with its effects, including situations of taking decisions about weight management. Stigma happens without awareness or attention from individuals to influence thoughts about weight management, making the decision making more difficult.

Neither of these clinically influential models, therefore, is useful in prompting clinicians to assess the impact of obesity stigma on a person's decision making about weight. This is of greater significance when set against evidence that sensitivity to obesity stigma is heightened for many individuals during clinical consultations [14,15, 40]. The attitudes of doctors and nurses with respect to obesity have been studied in many countries [16,41]. An overall conclusion is that the attitudes of these professionals reflect the weight biases and stereotypes of the populations they are drawn from [15]. Studies of patient perceptions indicate that this is a problem for patients who expect more sensitive treatment [23-25,40,42-44]. In the context of a consultation about weight therefore, obesity stigma has potential to undermine the relationship with the clinician as well as potentially heightening the decision making difficulty for the patient $[45,46]$. This may be a factor in the apparent high drop out for patients referred into weight management interventions $[17,47]$.

\subsection{Improving Decision Making}

How and why people make decisions has been studied across many contexts including health care $[48,49]$. It might be hoped that individuals carefully consider all the factors to arrive at a logical choice about what is going to do most good for them. In practice individuals are often unaware of how they make decisions and what factors influenced them. Generally a kind of "rule of thumb" thinking goes on that is selective with information. Decisions tend to be taken without much systematic thought, based on gut feelings and first impressions, and without being clear about influences. Each of these may bias against arriving at a good decision. Our study suggests decisions about weight management fit the general picture just described. In addition weight management is a particularly complex decision because of the extra thought pressures and influences highlighted.

In general decision making can be improved in a number of ways: by raising awareness that a decision making process is needed; by helping people to understand and evaluate the influences on their decision making; and by providing memory prompts and summaries that pull relevant information together to guide the decision process [48-50]. Practical interventions that address these issues are built around improving communication and rapport between patients and health professionals with decision aids as a facilitating tool [50-52]. Decisions about weight control could be improved with attention to each of these general points. Our study demonstrates people are not sufficiently aware of having to decide what to do. Awareness of the need for a further thought process is lost within the emotional resolving of ambivalent feelings about weight, size and personal responsibility. Furthermore, sensitivity to obesity stigma influences decision making with potential for biased evaluations and paradoxical inhibitions as regards good decision making. Finally, the amount of information and clear thinking necessary to arrive at a good decision about weight management is demanding. A decision aid that supports patients with decisions about weight management would be useful. It also has potential to foster the rapport, relationship and concordance with the clinician-all of which may be undermined otherwise.

\subsection{Conclusion}

Obesity stigma may add a mix of negative stereotypes and anticipations to thoughts within a typically selective, unsystematic thinking process in deciding about weight management. Stigma contributes to a deeper ambivalence and difficulty in decision making. Decision aid interven- 
tions and training of health care staff in shared decision making provides a way forward.

\section{ACKNOWLEDGEMENTS}

We thank members of the user involvement group who advised on the study protocol. Ethical permission to conduct the study was received from Leeds East Local Research Ethics Committee. We thank Ms Josie Messina who contributed to recruitment and data collection. Particular thanks to the interview participants. IB led study design, data collection and analysis, and preparation of this article. AM contributed to data analysis, interpretation and approval of the final article. This study was in receipt of funding from the Collaboration for Leadership in Applied Health Research and Care for South Yorkshire (CLAHRC SY). CLAHRC SY acknowledges funding from the National Institute for Health Research (NIHR). The views and opinions expressed are those of the authors, and not necessarily those of the NHS, the NIHR or the Department of Health.

\section{REFERENCES}

[1] World Health Organisation (2000) Obesity: Preventing and managing the global epidemic. WHO, Geneva.

[2] Hu, J. (2008) Obesity epidemiology. Oxford University Press, New York. doi:10.1093/acprof:oso/9780195312911.001.0001

[3] Foresight (2007) Tackling obesities: Future choices. Government Office for Science, London.

[4] US Department of Health and Human Services (2001) The surgeon-general's call to action to prevent and decrease overweight and obesity. US GPO, Washington.

[5] National Institutes of Health \& NHLBI (1998) Clinical guidelines on the identification, evaluation and treatment of overweight and obesity in adults-The evidence report. Obesity Research, 6, 1-209. doi:10.1002/j.1550-8528.1998.tb00681.x

[6] NHMRC (2003) Clinical practice guidelines for the management of overweight and obesity in adults. National Health and Medical Research Council, Canberra.

[7] NICE (2006) Obesity: Guidance on the prevention, identification, assessment and management of overweight and obesity in adults and children. National Institute for Health and Clinical Excellence, London.

[8] SIGN (2010) Management of obesity: A national clinical guideline. Scottish Intercollegiate Guidelines Network; Edinburgh.

[9] Douketis, J., Macie, C., Thabane, L. and Williamson, D. (2005) Systematic review of long-term weight loss studies in obese adults: Clinical significance and applicability to clinical practice. International Journal of Obesity, 29, 1153-1167. doi:10.1038/sj.ijo.0802982

[10] Loveman, E., Frampton, G., Shepherd, J.P., Cooper, K., Bryant, J. and Welch, K. (2011) The clinical effectiveness and cost effectiveness of long-term weight management schemes for adults: A systematic review. Health Technology Assessment, 15, 1-182.
[11] Karasu, S. and Karasu, T. (2010) The gravity of weight: A clinical guide to weight loss and maintenance. American Psychiatric Publishing, Washington.

[12] TOS Obesity as a Disease Writing Group (2008) Obesity as a disease. Obesity, 16, 161-177.

[13] Akabas, S., Lederman, S. and Moore, B. (2012) Textbook of obesity: Biological, psychological and cultural influences. John Wiley \& Sons, Chichester.

[14] Brownell, K., Puhl, R., Schwartz, M. and Rudd, L. (2005) Weight bias: Nature, consequences and remedies. Guilford Press, New York.

[15] Puhl, R. and Heuer, C. (2009) The stigma of obesity: A review and update. Obesity, 17, 941-964. doi:10.1038/oby.2008.636

[16] Sabin, J., Marini, M. and Nosek, B. (2012) Implicit and explicit anti-fat bias among a large sample of medical doctors by BMI, race/ethnicity and gender. PLoS One, 7, e48448. doi:10.1371/journal.pone.0048448

[17] Finley, C., Barlow, C. and Greenway, F. (2007) Retention rates and weight loss in a commercial weight loss program. International Journal of Obesity, 31, 292-298. doi:10.1038/sj.ijo.0803395

[18] Green, J. and Thorogood, N. (2009) Qualitative methods for health research. Sage, London.

[19] Ritchie, J. and Lewis, J. (2003) Qualitative research practice. Sage, London.

[20] Mays, N. and Pope, C. (2000) Assessing quality in quailtative research. British Medical Journal, 320, 50-52. doi:10.1136/bmj.320.7226.50

[21] The PLoS Medicine Editors (2007) Qualitative research: Understanding patients' needs and experiences. PLoS Medicine, 4, 1283-1284.

[22] Relton, C., Bissell, P. and Smith, C. (2011) South Yorkshire Cohort: A “cohort trials facility" study of health and weight_Protocol for the recruitment phase. BMC Public Health, 11, 640. doi:10.1186/1471-2458-11-640

[23] Brown, I. and Gould, G. (2011) Decisions about weight management: A synthesis of qualitative studies of obesity. Clinical Obesity, 1, 99-109. doi:10.1111/j.1758-8111.2011.00020.x

[24] Psarou, K. and Brown, I. (2010) Patients’ experiences of prescribed anti-obesity drugs and perceptions of support from primary care: A qualitative study. Primary Health Care Research and Development, 11, 250-259. doi:10.1017/S1463423610000083

[25] Brown, I., Thompson, J., Tod, A. and Jones, G. (2006) Primary care support for tackling obesity: Qualitative study of the perceptions of obese patients. British Journal of General Practice, 56, 666-672.

[26] Gibbs, G. (2002) Qualitative data analysis: Explorations with NVivo. Open University Press, Buckingham.

[27] Richie, J., Spencer, L. and O’Connor, W. (2003) Carrying out qualitative analysis. In: Richie, J. and Lewis, J. Eds., Qualitative Research Practice, Sage, London.

[28] Thomas, S.L., Hyde, J., Karunaratne, A., Herbert, D. and Komesaroff, P.A. (2008) Being “fat” in today’s world: A 
qualitative study of the lived experiences of people with obesity in Australia. Health Expectations, 11, 321-330. doi:10.1111/j.1369-7625.2008.00490.x

[29] Brown, I., Thompson, J. and Jones G. (2006) Obesity, stigma and quality of life. International Journal of Interdisciplinary Social Sciences, 1, 169-178.

[30] Granberg, E. (2006) “Is that all there is?” possible selves, self-change, and weight loss. Social Psychology Quarterly, 69,109-126. doi:10.1177/019027250606900201

[31] Blixen, C., Singh, A. and Thacker, H. (2006) Values and beliefs about obesity and weight reduction among African-American and Caucasian women. Journal of Transcultural Nursing, 17, 290-297. doi:10.1177/1043659606288375

[32] Crocker, J., Cornwell, B. and Major, B. (1993) The stigma of overweight-Affective consequences of attributional ambiguity. Journal of Personality and Social Psychology, 64, 60-70. doi:10.1037/0022-3514.64.1.60

[33] Crandall, C. and Martinez, R. (1996) Culture, ideology and anti-fat attitudes. Personality and Social Psychology Bulletin, 22, 1165-1176. doi:10.1177/01461672962211007

[34] Smith, E., Hay, P., Campbell, L. and Trollor, J. (2011) A review of the association between obesity and cognitive function across the lifespan: Implications for novel approaches to prevention and treatment. Obesity Reviews, 12, 740-755.

[35] Baronowski, T., Cullen, W., Nicklas, T., Thompson, D. and Baronowski, J. (2003) Are current health behavioural change models helpful in guiding prevention of weight gain efforts? Obesity Research, 11, S23-S43. doi:10.1038/oby.2003.222

[36] Prochaska, J. and Velicer, W. (1997) The transtheoretical model of health behaviour change. American Journal of Health Promotion, 12, 38-48. doi:10.4278/0890-1171-12.1.38

[37] West, R. (2005) Time for a change: Putting the Transtheoretical (stages of change) model to rest. Addiction, 100, 1036-1039. doi:10.1111/j.1360-0443.2005.01139.x

[38] Ajzen, I. (1991) The theory of planned behaviour. Organizational Behavior and Human Decision Processes, 50, 179-211. doi:10.1016/0749-5978(91)90020-T

[39] Armitage, C. and Conner, M. (2001) Efficacy of the theory of planned behaviour. A meta-analytic review. British Journal of Social Psychology, 40, 471-499. doi:10.1348/014466601164939

[40] Puhl, R. and Brownell, K. (2006) Confronting and coping with weight stigma: An investigation of overweight and obese adults. Obesity, 14, 1802-1815. doi:10.1038/oby.2006.208
[41] Brown, I. (2006) Nurses' attitudes towards adult patients who are obese: Literature review. Journal of Advanced Nursing, 53, 221-232. doi:10.1111/j.1365-2648.2006.03718.x

[42] Ogden, J., Bandara, I., Cohen, H., Farmer, D., Hardie, J. and Minas, H. (2001) General practitioners' and patients' models of obesity: Whose problem is it? Patient Education \& Counselling, 44, 227-233. doi:10.1016/S0738-3991(00)00192-0

[43] Wadden, T.A., Anderson, D.A., Foster, G.D., Bennett, A., Steinberg, C. and Sarwer, D.B. (2000) Obese women's perceptions of their physicians' weight management attitudes and practices. Archives of Family Medicine, 9, 854860. doi:10.1001/archfami.9.9.854

[44] Greiner, K.A., Born, W., Hall, S., Hou, Q., Kimminau, K.S. and Ahluwalia, J.S. (2008) Discussing weight with obese primary care patients: Physician and patient perceptions. Journal of General Intern Medicine, 23, 581587. doi:10.1007/s11606-008-0553-9

[45] Malterud, K. and Ulriksen, K. (2011) Obesity, stigma and responsibility in health care: A synthesis of qualitative studies. International Journal of Qualitative Stud Health Well-Being, 6, e8404. doi:10.3402/qhw.v6i4.8404

[46] Brown, I. and Thompson, J. (2007) Primary care nurses' attitudes, beliefs and own body size in relation to obesity management. Journal of Advanced Nursing, 60, 535-543. doi:10.1111/j.1365-2648.2007.04450.x

[47] Huisman, S., Maes, S., de Gucht, V., Chatrou, M. and Haak, H. (2010) Low goal ownership predicts drop-out from a weight intervention study in overweight patients with type 2 diabetes. International Journal of Behavioural Medicine, 17, 176-181. doi:10.1007/s12529-009-9071-3

[48] Edwards, A. and Elwyn, G., Eds. (2009) Shared decisionmaking in health care: Achieving evidence-based patient choice. 2nd Edition, Oxford University Press, Oxford.

[49] DaSilva, D. (2012) Helping people share decision making. The Health Foundation.

[50] Stacy, D., Bennett, C., Barry, M., Col, N., Eden, K.B., Holmes-Rovner, M. and Llewellyn-Thomas, H. (2011) Decision aids for people facing health treatment or screening decisions. Cochrane Database of Systematic Reviews, 3.

[51] Rollnick, S., Miller, W. and Butler, C. (2008) Motivational interviewing in health care: Helping patients change Behaviour. Guildford Press, London.

[52] Silverman, J., Kurtz, S. and Draper, J. (2004) Skills for communicating with patients. Radcliffe Publishing, London. 